\title{
Structural, Electronic and Magnetic Properties of a Few Nanometer-Thick Superconducting $\mathrm{NdBa}_{2} \mathrm{Cu}_{3} \mathrm{O}_{7}$ Films
}

\author{
Marco Moretti Sala ${ }^{1, *,+}+{ }^{\circ}$, Marco Salluzzo ${ }^{2,+}$, Matteo Minola ${ }^{3,+}{ }^{\circledR}$, Gabriella Maria De Luca ${ }^{2,4}$, \\ Greta Dellea ${ }^{1}$, Vesna Srot ${ }^{3}$, Yi Wang ${ }^{3}$, Peter A. van Aken ${ }^{3}{ }^{-}$, Matthieu Le Tacon ${ }^{5}$, \\ Bernhard Keimer ${ }^{3}$, Claudia Dallera ${ }^{1}$, Lucio Braicovich ${ }^{1}$ and Giacomo Ghiringhelli ${ }^{1,6, *(D)}$ \\ 1 Dipartimento di Fisica, Politecnico di Milano, Piazza Leonardo da Vinci 32, I-20133 Milano, Italy; \\ greta.dellea@gmail.com (G.D.); claudia.dallera@polimi.it (C.D.); lucio.braicovich@polimi.it (L.B.) \\ 2 CNR-SPIN, Complesso Monte Sant'Angelo-Via Cinthia, I-80126 Napoli, Italy; \\ marco.salluzzo@spin.cnr.it (M.S.); gabriellamaria.deluca@unina.it (G.M.D.L.) \\ 3 Max Planck Institut für Festkörperforschung, Heisenbergstrasse 1, D-70569 Stuttgart, Germany; \\ m.minola@fkf.mpg.de (M.M.); V.Srot@fkf.mpg.de (V.S.); y.wang@fkf.mpg.de (Y.W.); \\ p.vanaken@fkf.mpg.de (P.A.v.A.); B.Keimer@fkf.mpg.de (B.K.) \\ 4 Dipartimento di Fisica "E. Pancini", Università Degli Studi di Napoli "Federico II", \\ Complesso Monte Sant'Angelo-Via Cinthia, I-80126 Napoli, Italy \\ 5 Institute for Quantum Materials and Technologies, Karlsruhe Institute of Technology, \\ Hermann-v.-Helmholtz Platz 1, 76344 Eggenstein-Leopoldshafen, Germany; matthieu.tacon@kit.edu \\ 6 CNR-SPIN, Dipartimento di Fisica, Politecnico di Milano, Piazza Leonardo da Vinci 32, I-20133 Milano, Italy \\ * Correspondence: marco.moretti@polimi.it (M.M.S.); giacomo.ghiringhelli@polimi.it (G.G.) \\ + These authors contributed equally to this work.
}

Received: 30 March 2020; Accepted: 21 April 2020; Published: 24 April 2020

\begin{abstract}
Epitaxial films of high critical temperature $\left(T_{c}\right)$ cuprate superconductors preserve their transport properties even when their thickness is reduced to a few nanometers. However, when approaching the single crystalline unit cell (u.c.) of thickness, $T_{c}$ decreases and eventually, superconductivity is lost. Strain originating from the mismatch with the substrate, electronic reconstruction at the interface and alteration of the chemical composition and of doping can be the cause of such changes. Here, we use resonant inelastic $x$-ray scattering at the $\mathrm{Cu} L_{3}$ edge to study the crystal field and spin excitations of $\mathrm{NdBa}_{2} \mathrm{Cu}_{3} \mathrm{O}_{7-x}$ ultrathin films grown on $\mathrm{SrTiO}_{3}$, comparing 1,2 and 80 u.c.-thick samples. We find that even at extremely low thicknesses, the strength of the in-plane superexchange interaction is mostly preserved, with just a slight decrease in the 1 u.c. with respect to the 80 u.c.-thick sample. We also observe spectroscopic signatures for a decrease of the hole-doping at low thickness, consistent with the expansion of the $c$-axis lattice parameter and oxygen deficiency in the chains of the first unit cell, determined by high-resolution transmission microscopy and x-ray diffraction.
\end{abstract}

Keywords: unit cell-thick films; superconductivity; resonant inelastic x-ray scattering

\section{Introduction}

The quest for miniaturization of technological devices has pushed the development of nanotechnology, a field of applied research that deals with materials whose properties are intimately related to, and strongly dependent on their lateral size and shape. Ultimately, when the process of miniaturization is taken to the extreme, the resulting material could be made by one or few of its building blocks, which in the case of crystalline solids is the compound unit cell, i.e., the smallest group 
of atoms that possesses the overall symmetry of the crystal. Within this framework, we investigated the properties of unconventional high-temperature superconductors in the form of films, with variable thicknesses down to the single unit cell, in order to monitor the evolution of their physical properties as a function of thickness and possibly isolate new phenomena.

High- $T_{\mathcal{C}}$ superconductors are materials that can have critical superconducting temperatures exceeding that of the boiling point of liquid nitrogen, and mostly include copper- and iron-based materials. They are "unconventional" superconductors, because their properties cannot be accounted for by the conventional Bardeen-Cooper-Schrieffer (BCS) theory of superconductivity [1]. High-temperature superconductivity was discovered in 1986 by Bednorz and Müller in an oxide of $\mathrm{Ba}$, La and $\mathrm{Cu}$ [2] and ever since physicists worldwide have been trying to explain its mechanism, which remains to date a subject of intense debate. Here, we focus our attention on ultrathin films of $\mathrm{NdBa}_{2} \mathrm{Cu}_{3} \mathrm{O}_{7-x}$, the member of a wide class of superconducting copper oxides $(\mathrm{Nd}, \mathrm{Y}) \mathrm{Ba}_{2} \mathrm{Cu}_{3} \mathrm{O}_{7-x}$ with critical temperatures above $90 \mathrm{~K}$ at ambient pressure. It is known that the transport properties of cuprate epitaxial films tend to degrade at extremely low thicknesses, in particular $T_{c}$ decreases and eventually superconductivity is lost [3-6]. Despite a quasi-perfect lattice matching with the substrates, that ensures high-quality crystalline growth above few nanometers, at very low coverage epitaxial strain might be imposing a non-negligible deformation to the cuprate structure [7]. Moreover, at the interface the electronic structure might feel the symmetry breaking and undergo some degree of reconstruction. Finally, and even more importantly, the actual chemical composition might be altered, leading to an effective doping of the $\mathrm{CuO}_{2}$ planes different from that of thicker films. A good evidence of the latter effect for the NBCO and YBCO families has been given in the recent literature, based on transport and structural studies $[4,6]$. What is missing is a spectroscopic analysis. Resonant inelastic $\mathrm{x}$-ray scattering is ideally suited for this purpose, as it probes the crystal field $(d d)$ excitations that are related to local coordination of the $\mathrm{Cu}$ ions [8-10], and the spin excitations (paramagnons) [11-15], whose dispersion is linked to the in-plane superexchange interaction. The latter is particularly significant for ultrathin films: is the antiferromagnetic short and medium range order preserved also in the single unit cell limit, where only $2 \mathrm{CuO}_{2}$ planes are present, or is the quasi-perfect two-dimensionality of the system an obstacle to such order? In order to correlate the changes in the crystallographic, electronic and magnetic properties of the films as a function of thickness, we carried out systematic structural and spectroscopic investigations. Note that RIXS measurements on low dimensional materials were reported previously in a superlattice consisting of 25 repetitions of $\mathrm{La}_{2} \mathrm{CuO}_{4} / \mathrm{LaAlO}_{3}$ building block layers, corresponding to an effective probed thickness of $33 \mathrm{~nm}$ [16]. Here, on the contrary, we probe a single layer of cuprate material, thus opening the way to the study of 2D-atomic materials by RIXS down to the single elementary unit cell.

\section{Materials and Methods}

Films of $\mathrm{NdBa}_{2} \mathrm{Cu}_{3} \mathrm{O}_{7-x}$ were grown on $\mathrm{TiO}_{2}$-terminated and (001)-oriented $\mathrm{SrTiO}_{3}$ substrates by high pure oxygen pressure diode sputtering [4,6]. In particular, we focus here on the 1, 2 and 80 u.c.-thick films: the growth rate was accurately calibrated by systematic x-ray reflectivity measurements on samples deposited with different deposition time, from 2 to $120 \mathrm{~min}$, and resulted to be 0.5 unit cells per minute. The uncertainty in the film thickness is only half unit cell, as confirmed by high-resolution scanning transmission electron microscopy (STEM) [17-19]. The quality of the crystalline structure is shown in the STEM images of Figure 1. In order to preserve the film quality, the epitaxial films were covered with a $2.4 \mathrm{~nm}$-thick amorphous layer of $\mathrm{NdBa}_{2} \mathrm{Cu}_{3} \mathrm{O}_{7-x}$ with the same nominal stoichiometry of the film, deposited at room temperature, which provides a barrier against external perturbation and prevents change in the oxygen stoichiometry. As shown in Figure 2 (left panel), the X-ray absorption spectra of an amorphous $(2.4 \mathrm{~nm}) \mathrm{NBCO}$ is characterized by a very small absorption due to copper, but does not give any significant contribution to the RIXS measurements at the peak of the $\mathrm{Cu} L_{3}$ absorption of the crystalline samples. Transport data shown in Figure 2 (right panel) show that a 1 u.c.-thick film is not superconducting, while superconductivity is observed 
already in the 2 u.c.-thick sample, with a reduced $T_{\mathcal{c}}$ of $30 \mathrm{~K}$, while the maximum $T_{\mathcal{c}}$ of $93 \mathrm{~K}$ is recovered already in 9 u.c.-thick samples, even when capped with the amorphous layer.

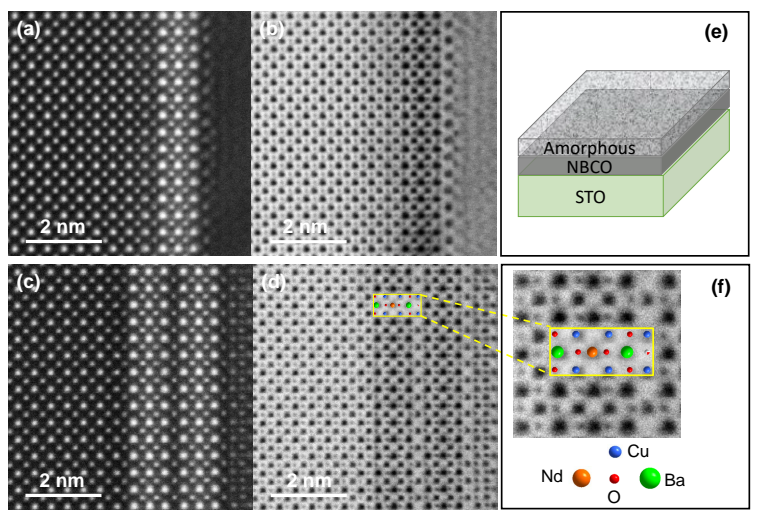

Figure 1. Scanning Transmission Electron Microscopy (STEM) images of epitaxial NBCO ultrathin films grown on STO with an overlay of the NBCO structural model. Images were obtained with the high-angle annular dark-field (HAADF) (panels a,c) and annular bright-field (ABF) (panels b,d) techniques. Panels $(\mathbf{a}, \mathbf{b})$ are for the 1 u.c. thick film, panels $(\mathbf{c}, \mathbf{d})$ for the 2 u.c. thick film. The details of atom position assignment is shown in panel (f). The scheme of the film growth structure is shown in panel (e).
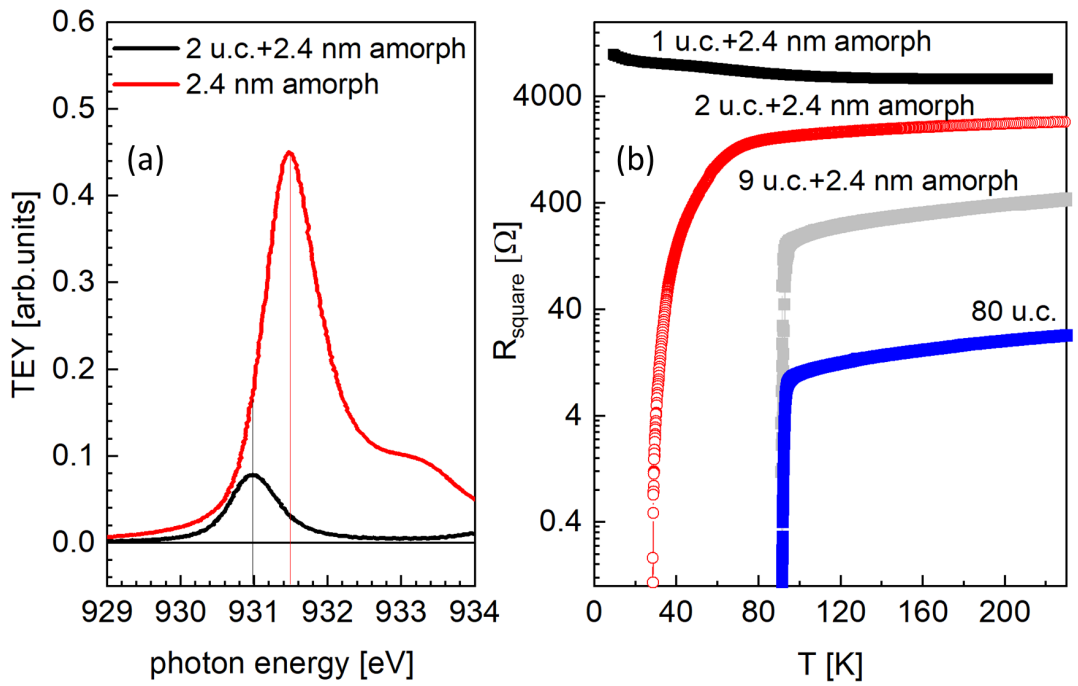

Figure 2. Panel (a) XAS measurements in total electron yield (TEY) mode of 2 u.c.-thick $\mathrm{NdBa}_{2} \mathrm{Cu}_{3} \mathrm{O}_{7-x}$ film covered with a $2.4 \mathrm{~nm}$-thick amorphous layer of the same material (red line) and of the $2.4 \mathrm{~nm}$-thick amorphous layer alone (black line). Panel (b) Resistivity measurements of 1 u.c. (black symbols), 2 u.c. (red symbols), 9 u.c. (gray symbols) -thick $\mathrm{NdBa}_{2} \mathrm{Cu}_{3} \mathrm{O}_{7-x}$ films covered with a 2.4 nm-thick amorphous layer of the same material and of 80 u.c.-thick $\mathrm{NdBa}_{2} \mathrm{Cu}_{3} \mathrm{O}_{7-x}$ film (blue symbols) with no capping layer.

X-ray diffraction (XRD) $\theta / 2 \theta$ measurements were performed by a conventional four-circle diffractometer (modified RIKAGU D-Max) equipped with a Cu-x-ray anode source. Transmission electron microscopy (TEM) measurements were carried out at the Stuttgart Center for Electron Microscopy of the Max Planck Institute for Solid State Research in Stuttgart (D). Resonant inelastic x-ray scattering (RIXS) measurements were performed at the ADRESS beam line [20] of the Swiss Light Source at the Paul Scherrer Institute (SLS-PSI) in Villigen (CH) using the SAXES [21] spectrometer. In order to obtain a reasonable count rate also on ultrathin films, the combined energy resolution was set to $190 \mathrm{meV}$ full-width-at-half-maximum at $931 \mathrm{eV}$, corresponding to the main peak in the $\mathrm{Cu} L_{3}$ edge x-ray absorption (XAS) profile of crystalline $\mathrm{NdBa}_{2} \mathrm{Cu}_{3} \mathrm{O}_{7-x}$. The polarization of the incoming photons 
was horizontal in the laboratory reference frame and parallel to the scattering plane. The scattering angle was set to $130^{\circ}$ in order to maximize the available momentum transfer. All measurements were performed at $T=10 \mathrm{~K}$, so that possible radiation damage effects were minimized.

\section{Results and Discussion}

$\mathrm{NdBa}_{2} \mathrm{Cu}_{3} \mathrm{O}_{7}$ in bulk form has an orthorhombic crystal structure with lattice parameters $a=3.87 \AA, b=3.91 \AA$ and $c=11.74 \AA$ and with $\mathrm{CuO}$ chains running along the $b$-axis separating bilayer units of $\mathrm{CuO}_{2}$ planes. XRD measurements show that above a threshold film thickness of about 60 u.c. the $\mathrm{NdBa}_{2} \mathrm{Cu}_{3} \mathrm{O}_{7-x}$ film is fully relaxed and its lattice parameters match those of bulk samples. Below 60 u.c., instead, $\mathrm{NdBa}_{2} \mathrm{Cu}_{3} \mathrm{O}_{7-x}$ films are subject to a small in-plane tensile strain and adopt a tetragonal crystal structure with in-plane lattice parameters perfectly matched with the STO substrate $(a=b=3.905 \AA)$ and short $\mathrm{CuO}$ chains running along both $\mathrm{a}$ and $\mathrm{b}$ axis. This suggests a clean pseudomorphic growth during the epitaxial deposition. Nevertheless, despite the sign of the strain, the $c$-axis increases as the film thickness decreases. For samples capped with the amorphous NBCO, the maximum value of the $c$-axis is $11.80 \AA$ in 1 u.c.-thick films. The combination of transport and x-ray diffraction results suggests a direct correlation between the $c$ lattice parameter and the doping level of $\mathrm{NdBa}_{2} \mathrm{Cu}_{3} \mathrm{O}_{7-x}$ : in particular, as for other high- $\mathrm{T}_{c}$ cuprates of the so-called 123-family, the $c$-axis expands when the oxygen stoichiometry in the chains decreases. Thus, the $c$-axis is one-to-one related to the oxygen stoichiometry and to the effective doping level of $\mathrm{NdBa}_{2} \mathrm{Cu}_{3} \mathrm{O}_{7-x}$. In our case, we estimate $x=0.6,0.5$ and about 0 for 1,2 and 80 u.c.-thick films, respectively, consistent with transport measurements and with the chemical analysis by high-resolution electron microscopy $[17,19]$.

The Figure $3 \mathbf{b}$ shows representative RIXS spectra of 1,2 and 80 u.c.-thick films at momentum transfer $q_{\|}=0.376$ r.l.u.. The acquisition time was 8 minutes for the 80 u.c.-thick sample and $30 \mathrm{~min}$ for the thinner ones, but data were normalized to the area of the spectrum in the energy region between 1 and $3 \mathrm{eV}$ energy loss because of the large absolute count-rate variation between the various samples. However, we first of all would like to emphasise the strong light-matter interaction that characterises RIXS, as opposed to the weak neutron-matter interaction in inelastic neutron scattering (INS), which makes RIXS suitable to study electronic, including magnetic, excitations in layers with thicknesses down to the single unit cell, in a reasonable amount of time.

The spectra in Figure 3 are characterized by a quasi-elastic line at zero energy loss, spin excitations around $0.3 \mathrm{eV}$ [22], while the most intense excitations at around $1.5 \mathrm{eV}$ are crystal-field (or dd) transitions $[10,21,22]$. The inset in the same figure shows that the contribution of the amorphous $\mathrm{NdBa}_{2} \mathrm{Cu}_{3} \mathrm{O}_{7}$ protective layer to the RIXS signal is negligible. Indeed, the XAS spectra of crystalline and amorphous $\mathrm{NdBa}_{2} \mathrm{Cu}_{3} \mathrm{O}_{7}$ show absorption resonances at distinct energies: this difference provides chemical sensitivity to RIXS and can be used to distinguish the contribution of $\mathrm{Cu}$ ions in different chemical environments.

First, we discuss the effect of the film thickness on $d d$ excitations, i.e., transitions between the crystal field split states of $\mathrm{Cu}^{2+}$. For bulk, insulating $\mathrm{NdBa}_{2} \mathrm{Cu}_{3} \mathrm{O}_{6}$, past RIXS studies allowed to determine with precision the following sequence of transition energies: the $3 z^{2}-r^{2}, x y$ and $z x / y z$ states of the $3 d^{9}$ hole are found at $1.98,1.52$ and $1.75 \mathrm{eV}$, respectively [10]. In the presence of doping, however, the corresponding peaks broaden and merge in an asymmetric, triangular feature, making the assignment of each individual final state impossible. The shape of the crystal-field excitation peak, therefore, reflects the degree of doping in the system: in our case, we observe a gradual evolution of its shape from very broad and asymmetric to rather sharp and symmetric as the film thickness is reduced, consistent with the variation of effective doping level invoked before. 

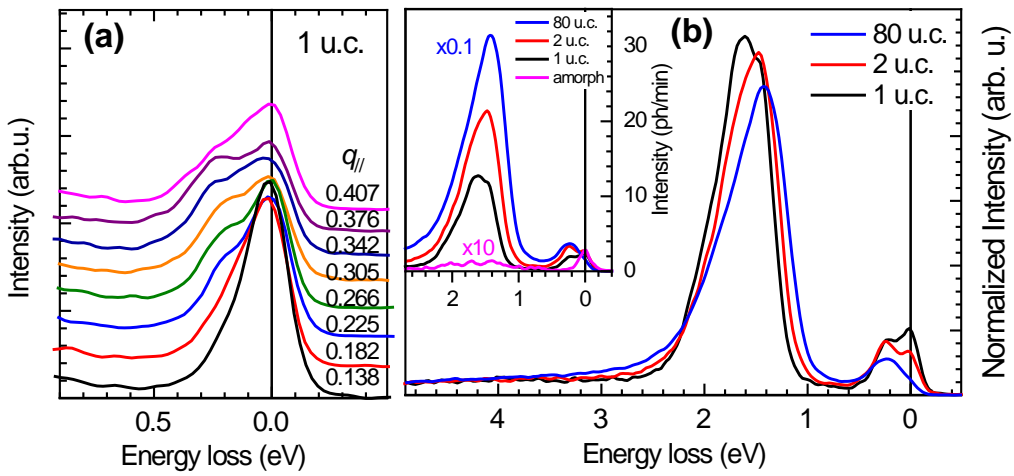

Figure 3. Panel (a) RIXS measurements of magnetic excitations in 1 u.c.-thick $\mathrm{NdBa}_{2} \mathrm{Cu}_{3} \mathrm{O}_{7-x}$ film as a function of momentum transfer $q_{\|}$. Panel (b) Comparison between the RIXS spectra at $q_{\|}=0.376$ r.l.u. of 1, 2 and 80 u.c.-thick $\mathrm{NdBa}_{2} \mathrm{Cu}_{3} \mathrm{O}_{7-x}$ films after normalization to the area of the $d d$ excitations. Also shown is the RIXS spectrum of a $2.4 \mathrm{~nm}$-thick amorphous layer of $\mathrm{NdBa}_{2} \mathrm{Cu}_{3} \mathrm{O}_{7-x}$, rescaled by a factor 10. The inset shows the same data normalized to the acquisition time.

We now turn to the analysis of the magnetic excitations, which in superconducting cuprates are heavily damped spin-excitations in the absence of long-range magnetic ordering and are, therefore, named paramagnons. Figure 3 a shows that magnetic excitations disperse as a function of the momentum transfer $q_{\|}$. The low-energy region of the RIXS spectra was fitted with two curves, an energy-resolution limited Gaussian for the elastic line and an asymmetric Voigt function for the paramagnon. Details of the fit for $q_{\|}=0.376$ r.l.u. are reproduced in Figure $4 \mathrm{a}-\mathrm{c}$. We note that the elastic line intensity increases as the film thickness is reduced, thus suggesting a growing non-resonant elastic scattering contribution from the substrate.

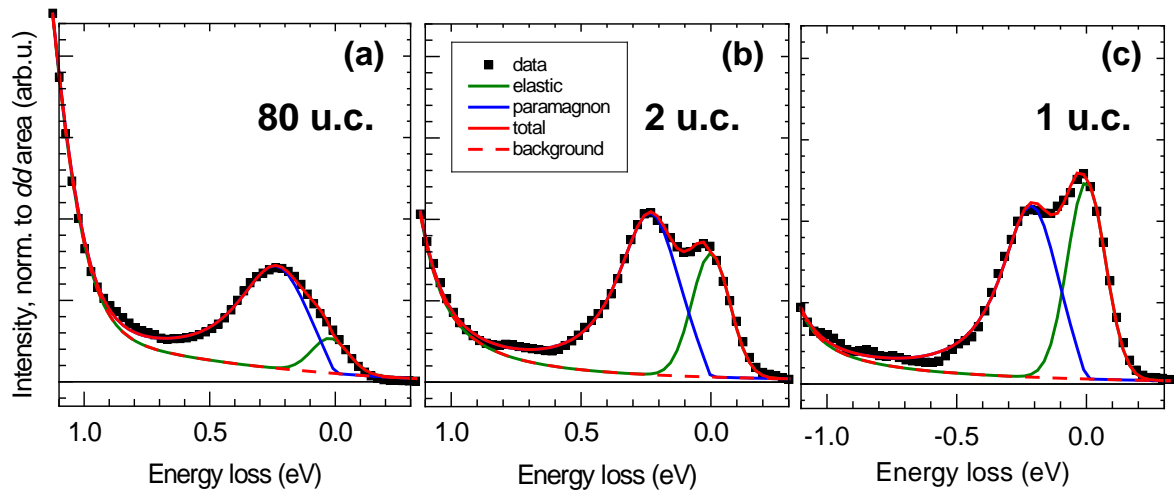

Figure 4. Panels (a-c): Fit to the RIXS spectra of 80,2 and 1 u.c-thick $\mathrm{NdBa}_{2} \mathrm{Cu}_{3} \mathrm{O}_{7-x}$ films at $q_{\|}=0.376$ r.l.u.

A summary of the main fitting parameters used to fit the RIXS data summarized in Figure 3 is provided in Figure 5. In particular, Figure 5a shows that the paramagnon energy slightly diminishes with the film thickness: the experimental data points are well fitted to the dispersion curve of optical magnons within the framework of a simple Heisenberg model, which provides the main in-plane (and out-of-plane) magnetic coupling(s) $J_{\|}$(and $J_{\perp}$, respectively). Note that, despite few authors have adopted a different interpretation of the spin excitations in doped cuprates [23], this approach is fully justified by a number of previous studies showing that, although damped, the spin excitations in doped cuprates remain well defined, preserve their spectral weight up to very high doping levels beyond optimal and perfectly reproduce the magnon dispersion observed in the AF insulators[12,13,24]. We find that $J_{\|}$evolves from $98 \mathrm{meV}$ for the 1 u.c.-thick film, to $110 \mathrm{meV}$ for the 2 u.c., to $114 \mathrm{meV}$ 
for the 80 u.c.-thick film (the out of plane coupling has been fixed at $7 \mathrm{meV}$ as in Ref. [25]). This little change can be consistent with the small variation of the in-plane lattice parameters vs. film thickness. On the other hand, Figure $5 \mathrm{~b}$ evidences a large variation of the paramagnon width at all transferred momenta: in particular, it is drastically reduced in the thinnest films as compared to the 80 u.c.-thick one. Again, this observation is consistent with the thickness dependence of the effective doping level and, in particular, with the evidence coming from transport measurements that the 1 u.c.-thick film is the least-doped sample. We note that in the data presented here, the mild tensile strain is playing only a secondary role in the determination of the thickness dependence of the crystal field and spin excitation energy. In other cases, e.g., for $\mathrm{CaCuO}_{2}$ [26] and $\mathrm{La}_{2} \mathrm{CuO}_{4}$ grown on different substrates [27], the larger strain leads to sizable changes of the hopping integrals that govern the $3 d$ orbital energy splitting and the superexchange parameter $J_{\|}$. On the contrary, here the dominant effect is the oxygen deficiency and correspondingly lower doping level of the ultrathin films, which leads to modifications of the RIXS spectra similar to those observed in bulk crystals, i.e., the broadening of the magnetic peak, with no or little variation of its energy position as a function of doping [28].
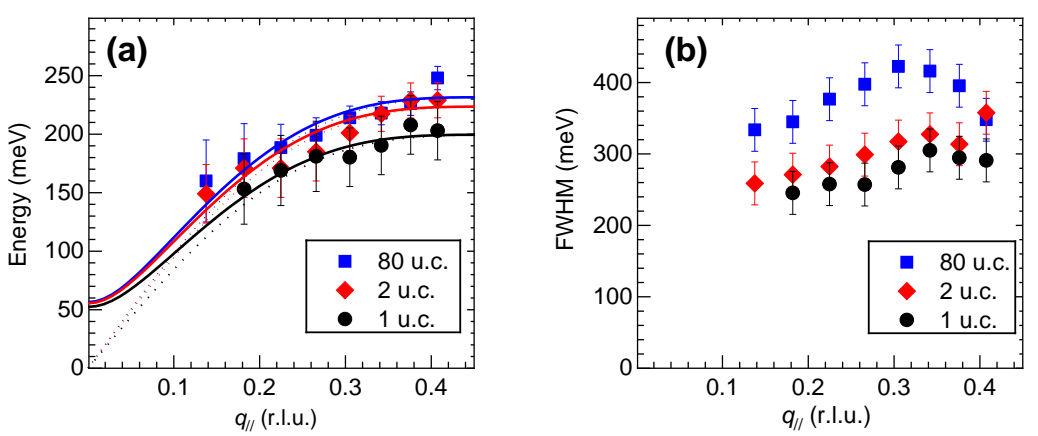

Figure 5. (a) Dispersion and (b) FWHM of magnetic excitations in 1 (black circles), 2 (red diamonds) and 80 (blue squares) u.c.-thick $\mathrm{NdBa}_{2} \mathrm{Cu}_{3} \mathrm{O}_{7-x}$ films. Continuous (dotted) lines show the corresponding optical (acoustic) magnetic branch used to fit the dispersion.

\section{Conclusions}

We were able to measure the RIXS response of $\mathrm{NdBa}_{2} \mathrm{Cu}_{3} \mathrm{O}_{7-x}$ films, with thicknesses down to the single unit cell, i.e., the smallest fundamental constituent of matter in crystalline form. This very fact proves the extreme sensitivity of RIXS in the study of nanometer-sized materials. Specifically, our measurements probe both magnetic and crystal-field (or $d d$ ) excitations and provide evidence of stoichiometry variations vs. thickness in nominally chemically equivalent films: in particular, we find that the effective doping of the $\mathrm{NdBa}_{2} \mathrm{Cu}_{3} \mathrm{O}_{7-x}$ films decreases as the film thickness is reduced. Our results are consistent with previous transport and structural investigations, thus providing a coherent picture of the electronic properties of ultrathin $\mathrm{NdBa}_{2} \mathrm{Cu}_{3} \mathrm{O}_{7-x}$ films. These results suggest that in cuprates, the degradation of superconducting properties when approaching the single unit cell thickness limit is related to the actual doping at the interface with the substrate rather than to the modification of the Mott-Hubbard physics in the $\mathrm{CuO}_{2}$ planes. Namely, the realization of almost ideal 2D superconductivity is not impeded by the supposed disruption of the strong antiferromagnetic background in the planes. On the contrary, short and medium range magnetic correlation persists down to 1 unit cell, leaving space for the Cooper pairing mediated by spin-fluctuation to be at play also in the extreme case of a single $\mathrm{CuO}_{2}$ plane [29], a scenario realized with half u.c.-thick $\mathrm{Bi}_{2} \mathrm{Sr}_{2} \mathrm{CaCu}_{2} \mathrm{O}_{8+x}$ [30] and recently with $\mathrm{Bi}_{2} \mathrm{Sr}_{2} \mathrm{CaCu}_{2} \mathrm{O}_{8+\delta}$ in the form of 1 unit cell thick flakes [31]. 
Author Contributions: G.G., L.B., M.L.T., B.K. and M.S. conceived the experiment; M.S. and G.M.D.L. grew and characterized the samples; M.M., M.S., G.D., G.G. performed the RIXS experiment; M.M., M.M.S. and G.G. analysed the RIXS spectra; V.S., Y.W. and P.A.v.A. performed the TEM measurements and analyzed them; M.M.S., C.D., M.S. and G.G. wrote the manuscript with inputs from all coauthors; M.L.T., B.K., G.G. supervised the project. All authors have read and agreed to the published version of the manuscript.

Funding: RIXS data were collected at the beam line ADRESS of the Swiss Light Source at the Paul Scherrer Institute (Switzerland) using the SAXES spectrometer jointly designed and built by the PSI and the Politecnico di Milano. M.M.S., C.D. and G.G. acknowledge support by project PRIN2017 “Quantum-2D” ID 2017Z8TS5B of the Ministry for University and Research (MIUR) of Italy. This project has received funding from the European Union's Horizon 2020 research and innovation program under grant agreement No. 823717-ESTEEM3.

Acknowledgments: C.D., M.M.S. and G.G. wish to recall and acknowledge their friendship and sincere admiration for Claudio Ferrero, a unique friend at ESRF. Claudio was the one person we were sure we would find in his office or around the ring, any time of the day and of the night, and would always have time for us, to chat or to discuss physics, or sometimes even to go for dinner... before returning to work again. He would always help us, in every aspect of work and life, switching effortlessly among half a dozen languages. He had the most brilliant mind, an astonishing memory, a tireless capability of tackling tens of problems in parallel, a correspondingly vast scientific production. He will always be an example to follow in many ways. The authors would like to acknowledge J. Pelliciari, Y. Huang and T. Schmitt for support during the RIXS experiment and R. Arpaia for useful discussions and critical reading of the manuscript.

Conflicts of Interest: The authors declare no conflict of interest.

\section{References}

1. Keimer, B.; Kivelson, S.A.; Norman, M.R.; Uchida, S.; Zaanen, J. From quantum matter to high-temperature superconductivity in copper oxides. Nature 2015, 518, 179-186. [CrossRef]

2. Bednorz, J.G.; Müller, K.A. Possible highTc superconductivity in the Ba-La-Cu-O system. Zeitschrift für Physik B Condensed Matter 1986, 64, 189-193. [CrossRef]

3. Triscone, J.M.; Karkut, M.G.; Antognazza, L.; Brunner, O.; Fischer, O. Y-Ba-Cu-O/Dy-Ba-Cu-O superlattices: A first step towards the artificial construction of high- $\mathrm{T}_{\mathrm{C}}$ superconductors. Phys. Rev. Lett. 1989, 63, 1016-1019. [CrossRef]

4. Salluzzo, M.; De Luca, G.; Marrè, D.; Putti, M.; Tropeano, M.; Scotti di Uccio, U.; Vaglio, R. Thickness effect on the structure and superconductivity of $\mathrm{Nd}_{1.2} \mathrm{Ba}_{1.8} \mathrm{Cu}_{3} \mathrm{O}_{z}$ epitaxial films. Phys. Rev. B 2005, 72, 134521. [CrossRef]

5. You, L.X.; Yurgens, A.; Winkler, D.; Lin, C.T.; Liang, B. Superconducting properties of ultrathin $\mathrm{Bi}_{2} \mathrm{Sr}_{2} \mathrm{CaCu}_{2} \mathrm{O}_{8+\mathrm{x}}$ single crystals. J. Appl. Phys. 2005, 98, 033913. [CrossRef]

6. Salluzzo, M.; De Luca, G.M.; Di Capua, R.; Gambardella, A.; Ristic, Z.; Vaglio, R. Electric field effect and superconducting-insulating transition in '123' cuprate superconductors. Supercond. Sci. Technol. 2009, 22, 034010. [CrossRef]

7. Arpaia, R.; Golubev, D.; Baghdadi, R.; Ciancio, R.; Dražić, G.; Orgiani, P.; Montemurro, D.; Bauch, T.; Lombardi, F. Transport properties of ultrathin $\mathrm{YBa}_{2} \mathrm{Cu}_{3} \mathrm{O}_{7-\delta}$ nanowires: A route to single-photon detection. Phys. Rev. B 2017, 96, 064525. [CrossRef]

8. Ghiringhelli, G.; Brookes, N.B.; Annese, E.; Berger, H.; Dallera, C.; Grioni, M.; Perfetti, L.; Tagliaferri, A.; Braicovich, L. Low Energy Electronic Excitations in the Layered Cuprates Studied by Copper $L_{3}$ Resonant Inelastic X-Ray Scattering. Phys. Rev. Lett. 2004, 92, 117406. [CrossRef]

9. Ghiringhelli, G.; Piazzalunga, A.; Wang, X.; Bendounan, A.; Berger, H.; Bottegoni, F.; Christensen, N.; Dallera, C.; Grioni, M.; Grivel, J.; et al. Crystal field and low energy excitations measured by high resolution RIXS at the L3 edge of Cu, Ni and Mn. Eur. Phys. J. Spec. Top. 2009, 169, 199-205. [CrossRef]

10. Moretti Sala, M.; Bisogni, V.; Aruta, C.; Balestrino, G.; Berger, H.; Brookes, N.B.; De Luca, G.M.; Di Castro, D.; Grioni, M.; Guarise, M.; et al. Energy and symmetry of dd excitations in undoped layered cuprates measured by $\mathrm{Cu} \mathrm{L}_{3}$ resonant inelastic x-ray scattering. New J. Phys. 2011, 13, 043026. [CrossRef]

11. Braicovich, L.; van den Brink, J.; Bisogni, V.; Moretti Sala, M.; Ament, L.J.P.; Brookes, N.B.; De Luca, G.M.; Salluzzo, M.; Schmitt, T.; Strocov, V.N.; et al. Magnetic Excitations and Phase Separation in the Underdoped $\mathrm{La}_{2-x} \mathrm{Sr}_{x} \mathrm{CuO}_{4}$ Superconductor Measured by Resonant Inelastic X-Ray Scattering. Phys. Rev. Lett. 2010, 104, 077002. [CrossRef] 
12. Le Tacon, M.; Ghiringhelli, G.; Chaloupka, J.; Moretti Sala, M.; Hinkov, V.; Haverkort, M.W.; Minola, M.; Bakr, M.; Zhou, K.J.; Blanco-Canosa, S.; et al. Intense paramagnon excitations in a large family of high-temperature superconductors. Nat. Phys. 2011, 7, 725-730. [CrossRef]

13. Dean, M.P.M.; Dellea, G.; Springell, R.S.; Yakhou-Harris, F.; Kummer, K.; Brookes, N.B.; Liu, X.; Sun, Y.J.; Strle, J.; Schmitt, T.; et al. Persistence of magnetic excitations in $\mathrm{La}_{2-x} \mathrm{Sr}_{x} \mathrm{CuO}_{4}$ from the undoped insulator to the heavily overdoped non-superconducting metal. Nat. Mater. 2013, 12, 1019-1023. [CrossRef]

14. Ivashko, O.; Shaik, N.E.; Lu, X.; Fatuzzo, C.G.; Dantz, M.; Freeman, P.G.; McNally, D.E.; Destraz, D.; Christensen, N.B.; Kurosawa, T.; et al. Damped spin excitations in a doped cuprate superconductor with orbital hybridization. Phys. Rev. B 2017, 95, 214508. [CrossRef]

15. Peng, Y.Y.; Huang, E.W.; Fumagalli, R.; Minola, M.; Wang, Y.; Sun, X.; Ding, Y.; Kummer, K.; Zhou, X.J.; Brookes, N.B.; et al. Dispersion, damping, and intensity of spin excitations in the monolayer $(\mathrm{Bi}, \mathrm{Pb})_{2}(\mathrm{Sr}, \mathrm{La})_{2} \mathrm{CuO}_{6+\delta}$ cuprate superconductor family. Phys. Rev. B 2018, 98, 144507. [CrossRef]

16. Dean, M.P.M.; Springell, R.S.; Monney, C.; Zhou, K.J.; Pereiro, J.; Bozovic, I.; Dalla Piazza, B.; Rønnow, H.M.; Morenzoni, E.; van den Brink, J.; et al. Spin excitations in a single $\mathrm{La}_{2} \mathrm{CuO}_{4}$ layer. Nat. Mater. 2012, 11, 850-854. [CrossRef]

17. Srot, V.; Wang, Y.; Minola, M.; Salluzzo, M.; De Luca, G.M.; Keimer, B.; van Aken, P.A. Direct Visualization and Image Simulations of Oxygen Sublattice Occupancy in Thin Cuprate Films. Microsc. Microanal. 2018, 24, 76-77. [CrossRef]

18. Srot, V.; Wang, Y.; Minola, M.; Salzberger, U.; Salluzzo, M.; De Luca, G.M.; Keimer, B.; van Aken, P.A. Combined imaging and analytical STEM of ultra-thin cuprate films. Microsc. Microanal. 2019, 25, 1750-1751. [CrossRef]

19. Srot, V.; Wang, Y.; Salzberger, U.; Fenk, B.; Kelsch, M.; Minola, M.; Salluzzo, M.; De Luca, G.M.; Keimer, B.; van Aken, P.A.; et al. Improved sample preparation of beam-sensitive ultra-thin cuprate films. Microsc. Microanal. 2019, 25, 686-687. [CrossRef]

20. Strocov, V.; Schmitt, T.; Flechsig, U.; Schmidt, T.; Imhof, A.; Chen, Q.; Raabe, J.; Betemps, R.; Zimoch, D.; Krempasky, J.; et al. High-resolution soft X-ray beamline ADRESS at the Swiss Light Source for resonant inelastic X-ray scattering and angle-resolved photoelectron spectroscopies. J. Synchrotron Radiat. 2010, 17, 631-643. [CrossRef]

21. Ghiringhelli, G.; Piazzalunga, A.; Dallera, C.; Trezzi, G.; Braicovich, L.; Schmitt, T.; Strocov, V.N.; Betemps, R.; Patthey, L.; Wang, X.; et al. SAXES, a high resolution spectrometer for resonant x-ray emission in the 400-1600 eV energy range. Rev. Sci. Instrum. 2006, 77, 113108. [CrossRef]

22. Fumagalli, R.; Braicovich, L.; Minola, M.; Peng, Y.Y.; Kummer, K.; Betto, D.; Rossi, M.; Lefrançois, E.; Morawe, C.; Salluzzo, M.; et al. Polarization-resolved $\mathrm{Cu} L_{3}$-edge resonant inelastic x-ray scattering of orbital and spin excitations in $\mathrm{NdBa}_{2} \mathrm{Cu}_{3} \mathrm{O}_{7-\delta}$. Phys. Rev. B 2019, 99, 134517. [CrossRef]

23. Benjamin, D.; Klich, I.; Demler, E. Single-Band Model of Resonant Inelastic X-Ray Scattering by Quasiparticles in High- $T_{\mathcal{C}}$ Cuprate Superconductors. Phys. Rev. Lett. 2014, 112, 247002. [CrossRef] [PubMed]

24. Le Tacon, M.; Minola, M.; Peets, D.C.; Moretti Sala, M.; Blanco-Canosa, S.; Hinkov, V.; Liang, R.; Bonn, D.A.; Hardy, W.N.; Lin, C.T.; et al. Dispersive spin excitations in highly overdoped cuprates revealed by resonant inelastic x-ray scattering. Phys. Rev. B 2013, 88, 020501. [CrossRef]

25. Peng, Y.Y.; Dellea, G.; Minola, M.; Conni, M.; Amorese, A.; Di Castro, D.; De Luca, G.M.; Kummer, K.; Salluzzo, M.; Sun, X.; et al. Influence of apical oxygen on the extent of in-plane exchange interaction in cuprate superconductors. Nat. Phys. 2017, 13, 1201-1206. [CrossRef]

26. Minola, M.; Hozoi, L.; Di Castro, D.; Felici, R.; Moretti Sala, M.; Tebano, A.; Balestrino, G.; Ghiringhelli, G.; van den Brink, J.; Braicovich, L. Measurement of the effect of lattice strain on magnetic interactions and orbital splitting in $\mathrm{CaCuO}_{2}$ using resonant inelastic x-ray scattering. Phys. Rev. B 2013, 87, 085124. [CrossRef]

27. Ivashko, O.; Horio, M.; Wan, W.; Christensen, N.B.; McNally, D.E.; Paris, E.; Tseng, Y.; Shaik, N.E.; Rønnow, H.M.; Wei, H.I.; et al. Strain-engineering Mott-insulating $\mathrm{La}_{2} \mathrm{CuO}_{4}$. Nat. Commun. 2019, 10, 786. [CrossRef]

28. Minola, M.; Dellea, G.; Gretarsson, H.; Peng, Y.Y.; Lu, Y.; Porras, J.; Loew, T.; Yakhou, F.; Brookes, N.B.; Huang, Y.B.; et al. Collective Nature of Spin Excitations in Superconducting Cuprates Probed by Resonant Inelastic X-Ray Scattering. Phys. Rev. Lett. 2015, 114, 217003. [CrossRef]

29. Scalapino, D.J. A common thread: The pairing interaction for unconventional superconductors. Rev. Mod. Phys. 2012, 84, 1383-1417. [CrossRef] 
30. Jiang, D.; Hu, T.; You, L.; Li, Q.; Li, A.; Wang, H.; Mu, G.; Chen, Z.; Zhang, H.; Yu, G.; et al. High-Tc superconductivity in ultrathin $\mathrm{Bi}_{2} \mathrm{Sr}_{2} \mathrm{CaCu}_{2} \mathrm{O}_{8+x}$ down to half-unit-cell thickness by protection with graphene. Nat. Commun. 2014, 5, 5708. [CrossRef]

31. Yu, Y.; Ma, L.; Cai, P.; Zhong, R.; Ye, C.; Shen, J.; Gu, G.D.; Chen, X.H.; Zhang, Y. High-temperature superconductivity in monolayer $\mathrm{Bi}_{2} \mathrm{Sr}_{2} \mathrm{CaCu}_{2} \mathrm{O}_{8+d}$. Nature 2019, 575, 156-163. [CrossRef] [PubMed]

(C) 2020 by the authors. Licensee MDPI, Basel, Switzerland. This article is an open access article distributed under the terms and conditions of the Creative Commons Attribution (CC BY) license (http:/ / creativecommons.org/licenses/by/4.0/). 